\title{
C syndrome
}

INSERM

\section{Source}

INSERM. (1999). Orphanet: an online rare disease and orphan drug data base. $\underline{C}$ syndrome. ORPHA:1308

C syndrome is a rare multiple congenital anomaly/intellectual disability syndrome characterized by trigonocephaly and metopic suture synostosis, dysmorphic facial features, short neck, skeletal anomalies, and variable intellectual disability. 